\title{
A functional study of the TatD DNases of Trueperella pyogenes and the conformational analysis of luteolin as an inhibitor
}

\author{
Zehui Zhang, Yinfeng Liang, Lihui Yu, Menghan Chen, Yuru Guo, Zhiruo Kang, Chenghu \\ Qu, Chunlian Tian, Dexian Zhang* and Mingchun Liu*
}

Key Laboratory of Livestock Infectious Diseases in Northeast China, Ministry of Education, College of Animal Science and Veterinary Medicine, Shenyang Agricultural University, Shenyang 110866, China;

1169175688@qq.com (Z.Z.); 949844003@qq.com (Y.L.); yuyang75060@163.com (L.Y.); 1499860870@qq.com (M.C.);1797667545@qq.com (Y.G.);1033001593@qq.com (Z.K.); 979662621@qq.com (C.Q.); chunliantian@syau.edu.cn (C.T.);

* Correspondence: zhangdx@syau.edu.cn (D.Z.); 1iumingchun@syau.edu.cn (M.L.)

Tel/fax: +86 02488487156 ;

\begin{abstract}
Trueperella pyogenes (T. pyogenes) can cause a variety of infections in animals and lead to economic loss in animal husbandry. TatD DNases are considered to be potential virulence factors in Plasmodium falciparum and Streptococcus pneumoniae. However, the function of TatD DNases in T. pyogenes is still unclear. Therefore, the aim of this study was to illustrate the function of TatD DNases of $T$. pyogenes (TpTatDs) and investigate whether luteolin is able to inhibit DNase function. The findings of our study are anticipated to be crucial to treating infections caused by $T$. pyogenes. Bioinformatic analysis has been used for the prediction of crucial functional residues of TpTatDs. The function of TpTatDs was investigated in the presence of divalent cations by hydrolyzing DNA with recombinant TatD proteins. Luteolin is a candidate nuclease inhibitor evaluated in our study. The interactions between luteolin and TpTatDs were tested using molecular docking analysis and surface plasmon resonance (SPR) assays. The inhibitory effect of luteolin on TpTatDs was analyzed by agarose gel electrophoresis. Two genes in the genome of $T$. pyogenes are suspected to encode TatD DNases. According to the length of their nucleotide sequences, they were named tatD960 and tatD825. Both of the TpTatDs, which are magnesium-dependent, were able to hydrolyze linear DNA and plasmids. In this study, we found through molecular docking analysis and SPR assays that luteolin can stably bind with TpTatDs. The gel assay revealed that luteolin can inhibit the DNase activity of TpTatDs. Our results indicated that TatD DNases from T. pyogenes are $\mathrm{Mg}^{2+}$-dependent DNases and exhibit DNA endonuclease activity. Moreover, luteolin reduced their DNA hydrolysis ability by decreasing the binding between TpTatDs and DNA.
\end{abstract}

Keywords: Trueperella pyogenes, TatD DNases, luteolin, DNA hydrolysis, molecular modeling, SPR.

\section{Introduction}

T. pyogenes is a gram-positive opportunistic pathogen commensally residing on mucous membranes. This pathogen can lead to mastitis, endometritis, cystitis, and orchitis by parasitizing the mucous membranes in many kinds of animals, such as swine, cattle and sheep [1,2]. T. pyogenes can cause 
great economic losses in animal husbandry because of the resulting decrease in meat and milk production and reproductive difficulties in infected food-producing animals [3-7]. Infection caused by T. pyogenes in humans is mostly related to occupational exposure and often occurs in immunosuppressed patients [8,9]. Potential manifestations of resulting inflammation in the body include local ulcers, pneumonia, sepsis urethritis, endocarditis and various abscesses [10-14]. Therefore, T. pyogenes is thought to be a zoonotic pathogen $[15,16]$.

TatD DNases exist widely in many kinds of organisms and are believed to perform several functions. For example, TatD DNases are capable of repairing their own DNA and are part of a quality control system that is intimately linked to the Tat export pathway in Escherichia coli [17-19]. On the other hand, TatD DNases are involved in programmed apoptosis in both Trypanosoma brucei and Phytophthora sojae, supporting the proliferation of these pathogens [20,21]. Moreover, TatD DNases from Plasmodium falciparum, African trypanosomes and Streptococcus pneumoniae are able to degrade neutrophil extracellular traps (NETs) and facilitate their escape from neutrophil immunity [2224]. However, the function of TatD DNase in T. pyogenes is still not reported.

Due to their wide range of sources and low toxicity, medicinal plant-derived native compounds are attracting great public interest. Luteolin (3',4',5,7-tetrahydroxyflavone), a natural flavonoid rich in a variety of plant products, exhibits antimicrobial activity by damaging bacterial cell membranes, cell walls and biofilms [25-28]. The main strategies by which luteolin affects bacterial proteins include binding to or interacting with proteins, decreasing the secretion of proteins, and inhibiting protein expression [29-32]. Our preliminary data suggest that luteolin can affect the expression of proteins and interfere with the normal processes of $T$. pyogenes [33]. Interestingly, luteolin also exerted a significant inhibitory effect on the production of the MATE protein in T. pyogenes by downregulating the expression of the MATE gene [34]. However, the effect of luteolin on TatD DNases still needs to be further investigated.

Therefore, our study aims to illustrate the function of TpTatDs and investigate the effect of luteolin on them. This study is anticipated to further reveal the potential application of luteolin as a potential DNase inhibitor for research on T. pyogenes.

\section{Results}

\subsection{Sequence characteristics of TpTatDs}

Genes encoding two TatD DNases with similar structural domains were found in the genomic DNA of T. pyogenes, and the encoded proteins were named TatD DNase 960 and TatD DNase 825 . A search of the Pfam protein family database using the $T$. pyogenes tat $D$ genes showed that the proteins encoded by the two genes belong to the TatD (PF01026) family, which is a family of proteins with TIM-barrel folds and conserved amino acid residues associated with DNase activity. Analysis using the SignalP 4.1 prediction tool showed that the two TpTatDs lacked signal peptides. By constructing a phylogenetic tree of 53 DNases from different bacteria, we found that 33 of the TatD DNases had similarity at the evolutionary level and were distinguished from other species of DNases, suggesting that the TatD DNase is highly evolutionarily conserved (Figure S1). The amino acid sequences of TpTatDs were compared with those of homologs from $S$. aureus, E. coli, and $S$. pneumoniae using Clustal W and DNAMAN for multiple sequence alignment, as shown in Figure 1A. TatD homologs were conserved in bacteria, and the sequence identity found by multiple sequence alignment of TatD DNase amino acid sequences from $S$. aureus, B anthracis, $S$. pneumoniae, E. coli and $T$. pyogenes was $35.76 \%$. The conserved sequences of TatD DNases from $T$. pyogenes and other bacteria may indicate that they may share similar functions. 
The amino acid sequences of TatD DNase 960 and TatD DNase 825 from $T$. pyogenes were used for SWISS-MODEL homology modeling to establish the possible three-dimensional structure of TatD DNases (Figure 1B,C). Based on sequence analysis, it can be predicted that His29, His31, His106, Glu157, His 194, His218 and Asp268 may be involved in binding metal ions in TatD DNase 960 and that Glu157, Glu266 and Asp268 may play a key role in the catalytic reaction (Figure 1B). In TatD DNase 825, His6, His8, His62, Gly91, His 132, His164 and Asp212 may be involved in binding metal ions, while Gly91 and Asp212 may be crucial in the catalytic reaction (Figure 1C).

A

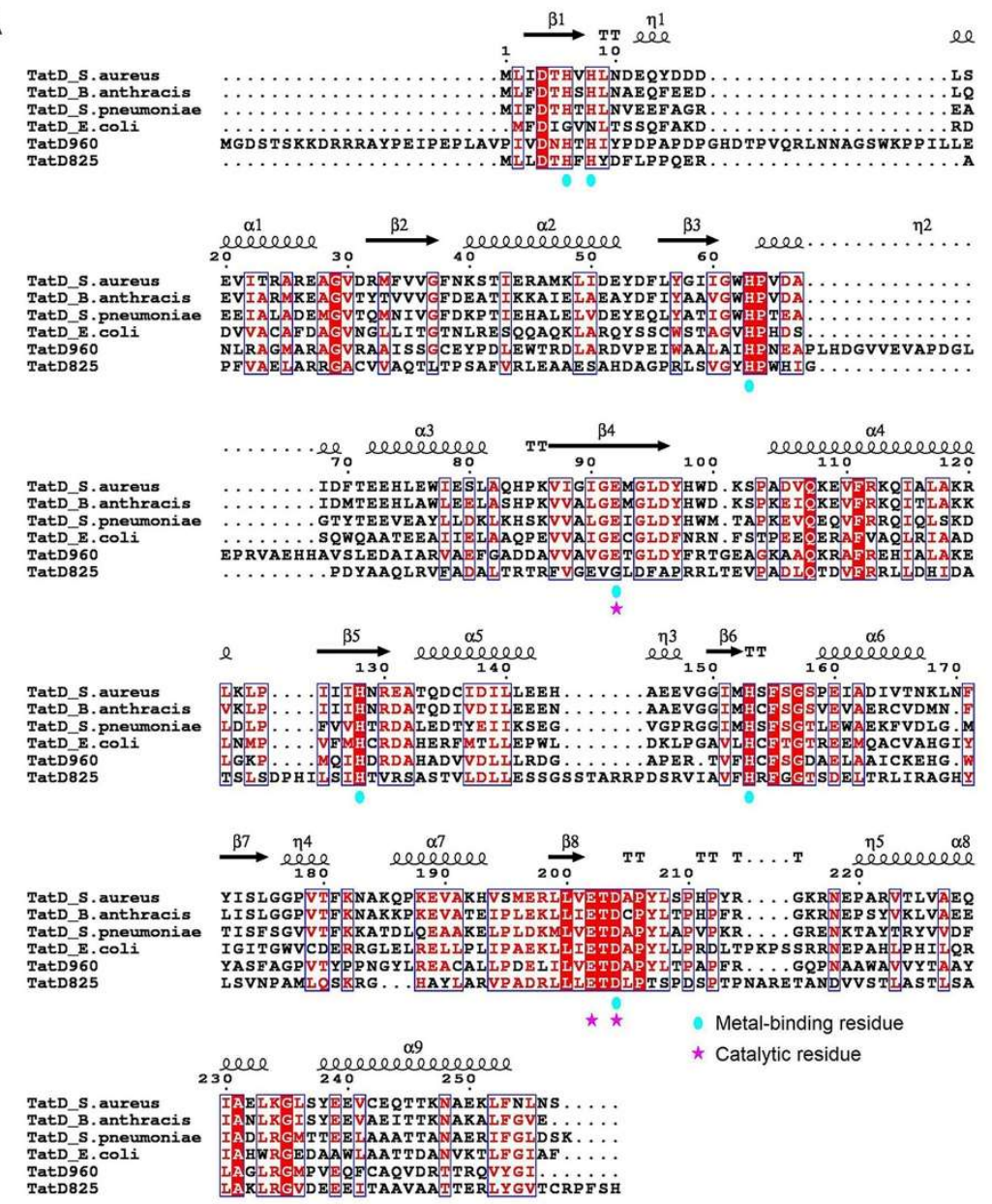

B

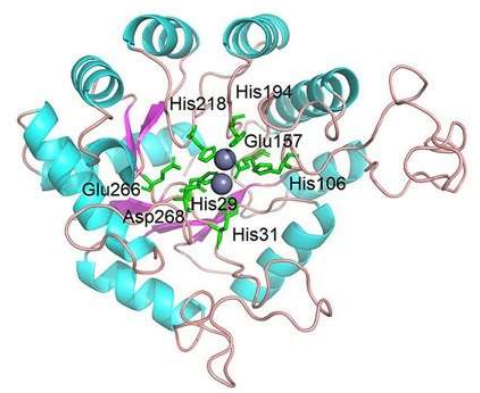

C

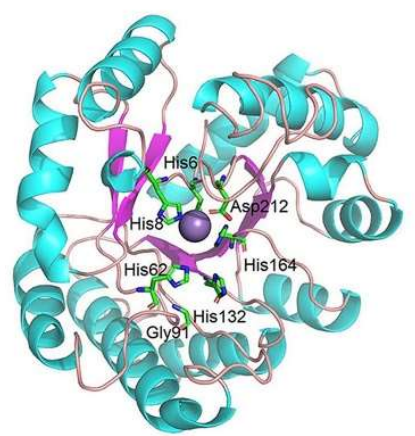

Figure 1. Amino acid sequences analysis and prediction of the three-dimensional structure of TatD DNases. (A) Amino acid sequences alignment of TatD DNases from $T$. pyogenes using ESPript with its homologs from $S$. aureus, $S$. pneumoniae, B. anthracis and E. coli. The conserved residues are highlighted in white on a red background. The similar residues are presented in red in blue boxes. The residues contributing to metal binding and catalysis are marked with blue circles and pink stars, respectively. (B) Structural modeling of TatD DNase 960 using SWISS-MODEL. The best match was found to be with $E$. coli TatD (PDB ID: 1YIX). (C)Structural modeling of TatD DNase 825 using SWISS-MODEL. The best match was found to be with E. coli TatD (PDB ID: 3GG7). Metal-binding and catalytic residues in TatD are highlighted in the drawing. 


\subsection{Preparation of recombinant TatD proteins}

To investigate the functional characteristics of TpTatDs, recombinant proteins were generated and purified. The molecular weights of TatD DNase 960 and TatD DNase 825 with His tags were $37 \mathrm{kDa}$ and $32 \mathrm{kDa}$, respectively (Figure 2A,B).
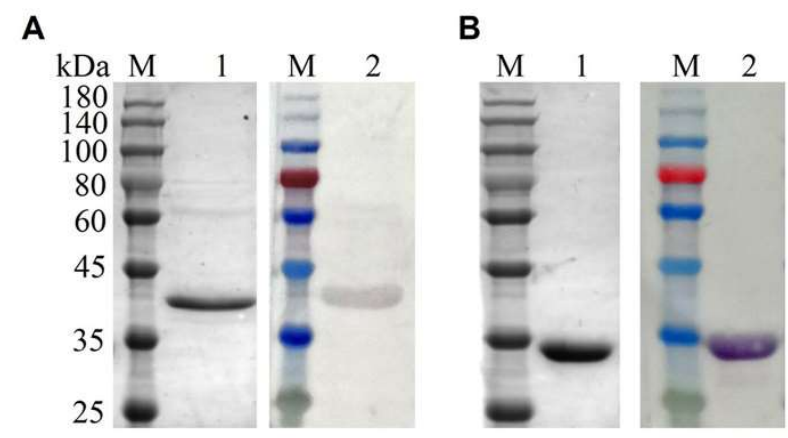

Figure 2. Purification of TpTatD recombinant proteins and identification using Western blot. (A) The purified TatD 960 recombinant protein $(37 \mathrm{kDa})$. (B) The purified TatD 825 recombinant protein $(32$ $\mathrm{kDa})$. Lane M: protein molecular weight standards; Lanes 1: SDS-PAGE analyses; Lanes 2: Western blot analyses.

\subsection{TpTatDs employ divalent metal ions for endonuclease activity}

An in-gel DNase activity assay showed that TatD DNase 960 and TatD DNase 825 have DNA degradation activity. Here, we demonstrated that divalent metal ions, including $\mathrm{Mg}^{2+}, \mathrm{Ca}^{2+}$ and $\mathrm{Ni}^{2+}$, promoted the DNA hydrolysis activity of TatD DNase 960 when recombinant TatD 960 proteins were incubated with DNA and various divalent metal ions (Figure 3A). Increasing the concentration of TatD DNase 960 or prolonging the reaction time improved the ability of TpTatD to hydrolyze DNA (Figure S2A,B). TatD DNase 960 hydrolyzed DNA in a $\mathrm{Mg}^{2+}$-dependent manner (Figure S2C,D). TatD DNase 960 also hydrolyzed circular DNA, and $\mathrm{Mg}^{2+}$ promoted the degradation of plasmid pBR322 by TatD DNase 960 (Figure S4). The effects of temperature and $\mathrm{pH}$ on the DNase activity of TatD DNase 960 were analyzed using a PicoGreen assay. The optimal reaction temperature for TatD DNase 960 was 37 ${ }^{\circ} \mathrm{C}$ (Figure 4A,B). To determine the $\mathrm{pH}$ dependence of DNase activity, recombinant TatD proteins were incubated with mouse liver DNA in PBS buffer with a $\mathrm{pH}$ ranging from 6 to 9.5. The recombinant TatD 960 protein showed DNase activity over a wide $\mathrm{pH}$ range, with the maximum DNase activity at pH 8.0 (Figure 4C).

Similar to that of TatD DNase 960, the catalytic activity of TatD DNase 825 showed metal ion dependence. $\mathrm{Mg}^{2+}$ and $\mathrm{Ca}^{2+}$ promoted DNA hydrolysis by TatD DNase 825 (Figure 3B). The DNase activity of TatD DNase 825 increased at higher concentrations of TatD DNase 825 and longer reaction times (Figure S3A,B). These results indicate that TatD DNase 825 is also a magnesium-dependent DNA endonuclease (Figures S3C,D, S4). The optimum reaction temperature for TatD DNase 825 was $37{ }^{\circ} \mathrm{C}$, and the maximum activity of TatD DNase 825 occurred at $\mathrm{pH} 7.5$ (Figure 4). The reaction conditions and enzymatic activity of TpTatDs were similar to those of DNase I, showing high industrial applicability (Figure 4C). 


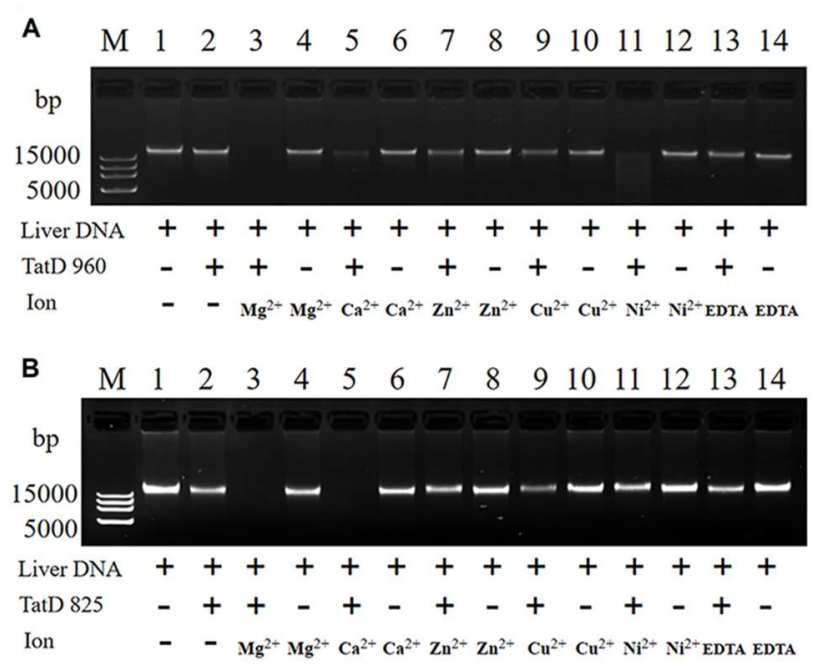

Figure 3. The effects of divalent metal cations on DNase activity of TpTatDs. Linear DNA was incubated in the absence (lanes 1, 4, 6, 8, 10, 12 and 14) or presence (lanes 2, 3, 5, 7, 9, 11 and 13) of TpTatDs and a variety of metal ions for $60 \mathrm{~min}$ at $37{ }^{\circ} \mathrm{C}$. DNA was visualized by agarose gel electrophoresis. The concentration of metal ions was10 mM. (A) The DNase activity of TatD DNase 960. (B) The DNase activity of TatD DNase 825.
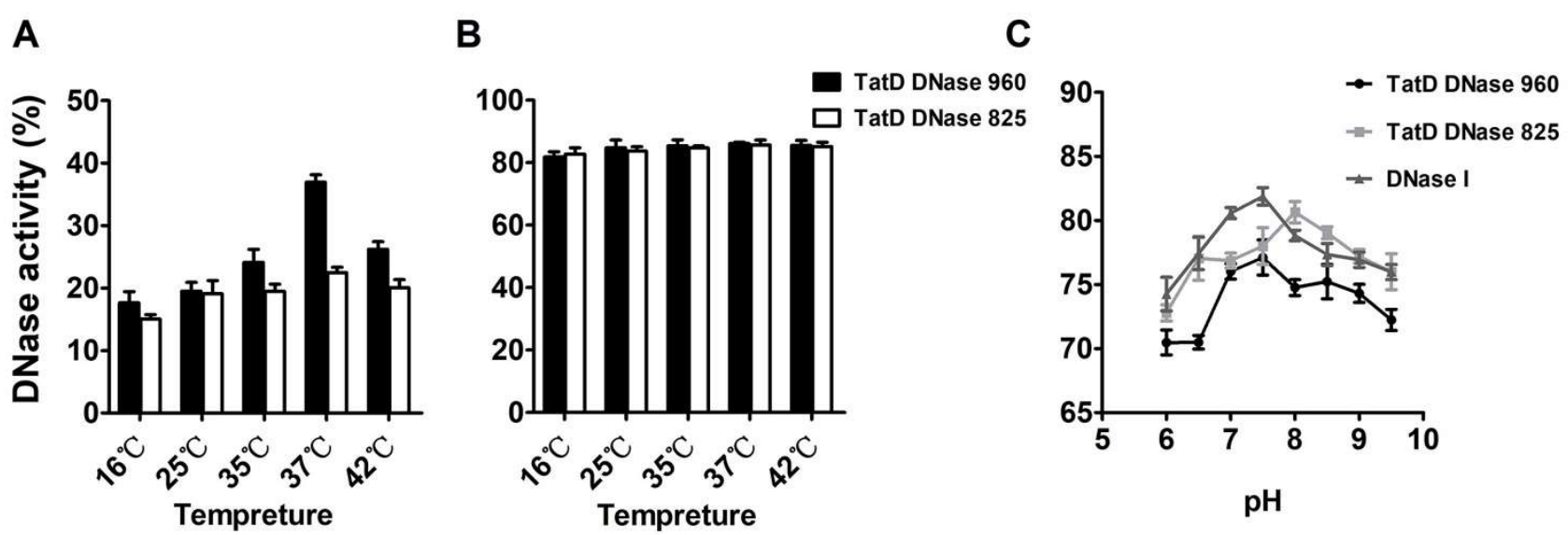

Figure 4. The effects of temperature and $\mathrm{pH}$ on DNase activity of TpTatDs. (A-B) Analysis of the effect of temperature on DNase activity of TpTatDs in the absence or presence of $\mathrm{Mg}^{2+}$ by PicoGreen assay. (C) Analysis of the effect of $\mathrm{pH}$ on DNase activity of TpTatDs in the presence of $\mathrm{Mg}^{2+}$ by PicoGreen assay. The reactions were performed in PBS buffer with $\mathrm{pH}$ ranging from 6 to 9.5. DNase I was used as a control.

\subsection{The interactions between luteolin and TpTatDs predicted by three-dimensional modeling}

We sought to verify whether luteolin could be an inhibitor of TatD DNase 960 and TatD DNase 825 . The preferential mechanism of binding between TatD DNase and luteolin was established by molecular kinetics simulations, which showed a MolDock score of $-109.598 \mathrm{kcal} / \mathrm{mol}$ for the TatD DNase 960 luteolin complex and a MolDock score of $-78.6995 \mathrm{kcal} / \mathrm{mol}$ for the TatD DNase 825 -luteolin complex (Figure 5A,B). These results suggest that the formation of TatD DNase-luteolin complexes involves 
hydrogen bonds, $\pi-\sigma$ interactions, anion- $\pi$ interactions and $\pi$-alkyl interactions, where luteolin is able to fall into the molecular binding pockets of TatD DNase 960 and TatD DNase 825 (Figure 5A,B).
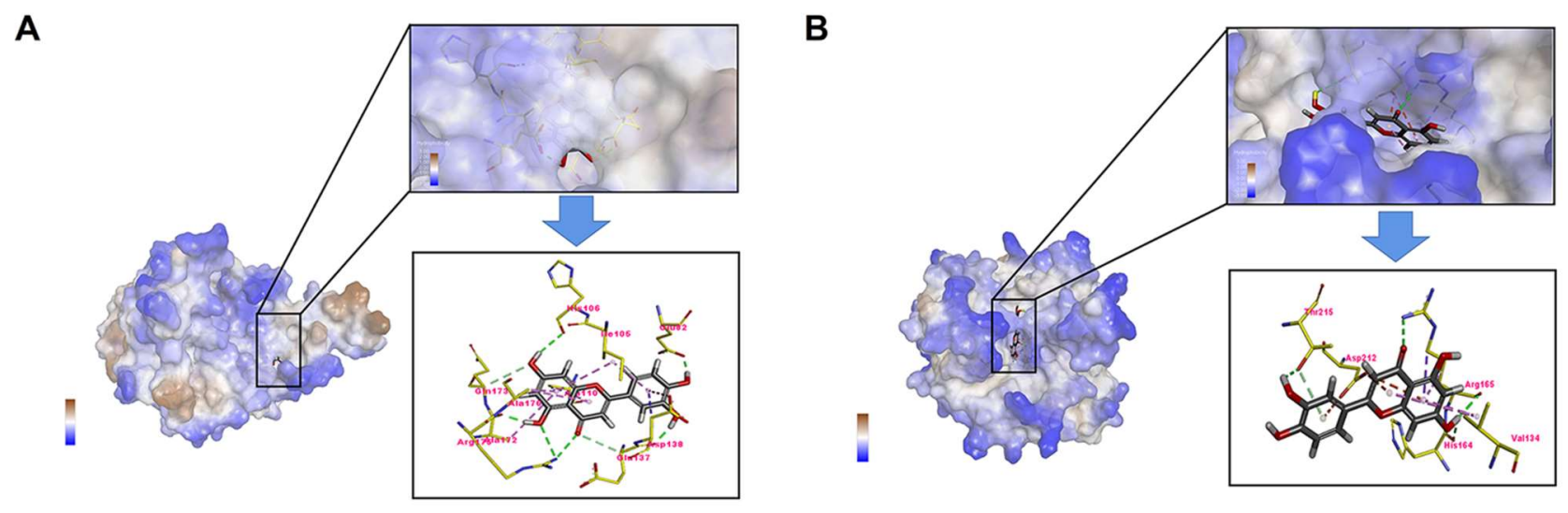

Figure 5. The interactions between luteolin and TatD DNases predicted by molecular modeling. (A) The model of interaction between luteolin and TatD DNase 960. (B) The model of interaction between luteolin and TatD DNase 825. The catalytic residues of interactions are highlighted in the enlarged view.

\subsection{Determination of affinities and kinetics of luteolin binding to TpTatDs using SPR technology}

We used a Biacore assay to further reveal the mechanism of action of luteolin binding with TpTatDs. The 1:1 binding model analysis indicated that luteolin was able to bind to TatD DNase 960 and TatD DNase 825 with associating kinetics and associated in a dose-dependent manner (Figure 6A,B). Small molecule protein affinity constants were typically $10^{-3}-10^{-6} \mathrm{M}$. Affinity fitting models showed that luteolin exhibited a moderate binding affinity for TatD DNase $960\left(\mathrm{~K}_{\mathrm{D}}=2.272 \times 10^{-4} \mathrm{M}\right)$ and TatD DNase $825\left(\mathrm{~K}_{\mathrm{D}}=1.816 \times 10^{-4} \mathrm{M}\right)$. These data corroborate the results of the molecular docking assay, suggesting that luteolin is capable of stably binding to TpTatDs and that their binding may impede TatD DNase action. 

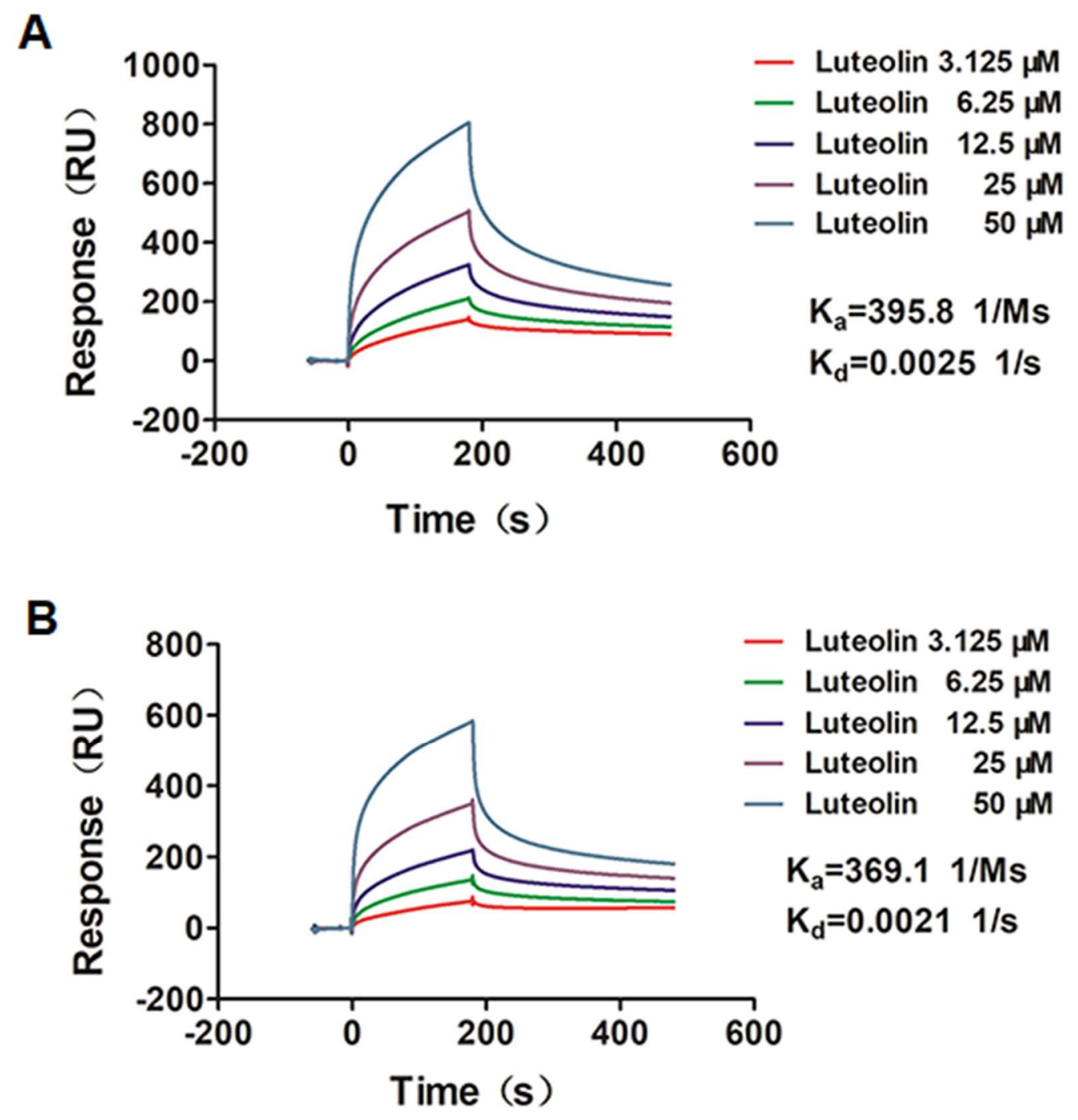

Figure 6. Binding of luteolin and TatD DNases in Biacore assays. Luteolin was tested in various concentrations $(3.125-50 \mu \mathrm{M})$. (A) Kinetic analysis of luteolin and TatD DNase 960. (B) Kinetic analysis of luteolin and TatD DNase 825 .

\subsection{Gel assay of TpTatD inhibition by luteolin}

We previously demonstrated that TatD DNases with $\mathrm{Mg}^{2+}$ can fully degrade DNA. When we incubated TpTatDs with different concentrations of luteolin $(0,1 / 8,1 / 4,1 / 2,1,2,4 \mathrm{MIC})$ in the presence of $\mathrm{Mg}^{2+}$, we found that luteolin effectively inhibited the activity of luteolin (Figure S5). When the concentration of luteolin was greater than $1 / 2 \mathrm{MIC}(39 \mu \mathrm{g} / \mathrm{mL})$, luteolin started to inhibit the hydrolysis of DNA by TpTatDs, and the inhibitory effect of luteolin on TatD DNase 960 and TatD DNase 825 showed concentration dependence.

\section{Discussion}

T. pyogenes synthesizes a number of proteases and nucleases that are thought to be associated with bacterial survival and pathogenicity [15]. TatD DNase is an evolutionarily conserved protein in bacteria, and T. pyogenes expressed two TatD DNases. Our data suggest that TatD DNase 960 and 
TatD DNase 825 of $T$. pyogenes are both $\mathrm{Mg}^{2+}$-dependent DNA endonucleases. Luteolin, a natural flavonoid, can bind to TpTatDs directly and inhibit their DNase activity by blocking their binding to DNA.

In this study, we constructed a phylogenetic tree of 53 DNases from bacteria and found that TatD DNases are evolutionarily conserved, suggesting that TatD DNases may have similar structures and functions among bacteria (Figure S1). The TatD protein was first identified to be encoded in the Tat operon in E. coli but is not directly linked to the transport function of the tat operon, and it is thought to be a DNase associated with the tat-associated quality control system [17,19]. We observed that TatD DNase 960 and TatD DNase 825 of T. pyogenes are both divalent metal ion-dependent DNases. $\mathrm{Mg}^{2+}$, $\mathrm{Ca}^{2+}$ and $\mathrm{Ni}^{2+}$ were able to promote the hydrolysis of DNA by TatD 960 , and $\mathrm{Mg}^{2+}$ and $\mathrm{Ca}^{2+}$ were able to promote the hydrolysis of DNA by TatD 825 (Figure 2A,B), similar to the function of the TatD DNase of E. coli [17]. Interestingly, in P. falciparum, the TatD DNase is considered to be a non-metal ion-dependent DNase; in Plasmodium knowlesi, $\mathrm{Mg}^{2+}$ promotes the enzymatic activity of the TatD DNase, and $\mathrm{Cu}^{2+}$ inhibits its hydrolysis of DNA; and hydrolysis mediated by TatD DNase of $S$. pneumoniae can be catalyzed by $\mathrm{Ca}^{2+}$ and $\mathrm{Co}^{2+}$ but inhibited by $\mathrm{Mg}^{2+}[22,24,35]$. The activity of the TatD DNase of P. falciparum is similar to that of DNase II, which is repressed by divalent metal ions; TpTatDs are divalent metal ion-dependent DNases, which have properties more similar to DNase I. These functional differences may be caused by differences in the amino acid residues associated with the binding of metal ions by TatD DNases or by mutations in the TIM barrel structure to perform a specific role. Resembling the TatD DNases of E. coli, S. aureus, B. anthracis and $S$ pneumoniae, TatD DNase 960 and TatD DNase 825 are both nucleic acid endonucleases with hydrolytic activity towards both double-stranded and circular DNA (Figure S4) [18,24,36,37]. TatD DNases from S. aureus have also been reported to have hydrolytic activity toward RNA [34].

The P. falciparum TatD DNase can degrade DNA in hosts when this pathogen escapes human immunity. When the body is infected with pathogens, neutrophils can produce reticular DNA structures called NETs, which can capture pathogens and kill them with antimicrobial peptides and other substances. The TatD DNase of $P$. falciparum can degrade NETs and help it escape neutrophil immunity. The TatD DNase of $P$. falciparum can also influence its pathogenicity and is a virulence factor [22]. The TatD DNase of $S$. pneumoniae, although lacking a signal peptide, can be secreted outside the bacterium by extracellular vesicles (EVs) and degraded to escape capture by NETs [24]. Studies on whether TatD DNases of T. pyogenes can mediate the ability to evade capture by NETs are still ongoing. TatD DNases may also be involved as a nuclease in the repair of DNA damage in the bacterium [18]. Therefore, the development of inhibitors of TatD DNases may limit the viability and pathogenicity of $T$. pyogenes, thus benefiting the treatment of clinically relevant diseases.

The effects of luteolin on crucial targets in pathogens have been widely reported. Luteolin has the ability to block the entry of coronaviruses into host cells and to specifically inhibit the major protease $3 \mathrm{CL}$ in the coronavirus infection cycle, thereby inhibiting viral infection $[38,39]$. The major flavonoid in honeysuckle, luteolin and its homologs can be used as influenza endonuclease inhibitors for antiinfluenza virus activity [40]. Metallo-beta-lactamase (MBL) is a kind of enzyme relevant to drug resistance in bacteria, and luteolin acts as an MBL inhibitor by occupying its active site [41]. Luteolin can also bind to histidine kinase through hydrogen bonding forces and $\pi-\pi$ stacking interactions, resulting in an inhibitory effect on Thermotoga maritime [42]. YbeY is a highly conserved bacterial RNase of Liberibacter asiaticus, and luteolin has inhibitory effects on key residues involved in its catalytic activity [43]. Studies at the molecular level have revealed that luteolin is a candidate broadacting enzyme inhibitor in the antimicrobial field. The binding or inhibitory effects of luteolin on specific targets have been extensively studied in pathogens, but those of luteolin in T. pyogenes remain 
unexplored. To determine the binding of luteolin to TatD DNases of T. pyogenes, the ligand-protein binding site was explored by molecular docking (Figure 5A,B). Our model suggests that luteolin can bind by forming a hydrogen bond with His106 of TatD DNase 960, which is predicted to be the key amino acid residue facilitating catalysis (Figures 1B,5A). Luteolin can also form hydrogen bonds with His 164 of TatD DNase 825, interacting with Asp212 in a anion- $\pi$ interactions (Figure 5B). His 164 is assumed to bind to metal ions to promote enzymatic reactions, and Asp212 is a key amino acid residue for TatD DNase 825 to perform its function in the degradation of DNA (Figure 1C). This observation suggests that the intermolecular forces between these important residues and luteolin are likely to competitively inhibit the binding between TatD DNases and DNA and impede enzymatic activity, resulting in reduced or the complete loss of TatD DNase enzymatic activity. This hypothesis is also supported by the luteolin-TatD DNase molecular interaction analysis and gel assay (Figures 6,S5). We recommend that medicinal chemists interested in studying TatD DNase inhibitors use luteolin as a tool compound. Our study demonstrates that luteolin is able to bind stably to TpTatDs and inhibit DNA degradation; unfortunately, the mechanism of the pathogenic effect of TpTatDs is unclear and is being explored in our further studies.

In conclusion, the following may be concluded: (i) TatD DNase 960 and TatD DNase 825 of $T$. pyogenes are DNA endonucleases with metal ion-dependent properties; and (ii) luteolin stably binds to TpTatDs and inhibits their DNase activity. Therefore, luteolin may be a potential inhibitor of TpTatDs, hindering the pathogenic development of $T$. pyogenes and providing a strategy to suppress bacterial infections in the clinic.

\section{Materials and Methods}

\subsection{Animals}

Female Kunming mice (6-8 weeks of age) were purchased from Changsheng Biological Technology co., Ltd (Shenyang, China; Permit NO. SYXK $<$ Liao $>2020-0001$ ) and maintained under specific pathogen-free conditions. The mouse livers were used to extract genomic DNA and every effort was made to minimize suffering. The animal study was reviewed and approved by Ethical Committee of Shenyang Agricultural University, China.

\subsection{T. pyogenes strains and culture}

The T. pyogenes isolate BMH06-3 was collected from dairy cattle in Liaoning, China, and identified by $16 S$ rRNA gene sequencing [33]. T. pyogenes strains were cultured on Mueller-Hinton agar (MHA, Solarbio, Beijing, China) containing $5 \%(\mathrm{v} / \mathrm{v})$ sheep blood (Solarbio, Beijing, China) in an incubator $\left(5 \% \mathrm{CO}_{2}\right)$ at $37{ }^{\circ} \mathrm{C}$ for $36 \mathrm{~h}$, and the colonies were inoculated in nutrient broth (NB, Solarbio, Beijing, China) supplemented with $8 \%(\mathrm{v} / \mathrm{v})$ fetal bovine serum (FBS, Gibco, Grand Island, USA). E. coli strains BL21 (DE3) (TransGen Biotech, Beijing, China) and Trans-1-T1 (TransGen Biotech Beijing, China) were cultured in Luria-Bertani broth (LB, Solarbio, Beijing, China) at $200 \mathrm{rpm}$ in a shaker or on LB agar plates supplemented with kanamycin $(50 \mu \mathrm{g} / \mathrm{ml})$ at $37^{\circ} \mathrm{C}$.

\subsection{Bioinformatic analysis of TpTatDs}

The gene sequences of TatD 960 and TatD 825 were searched in GenBank and matched with the genome of T. pyogenes isolate BMH06-3. The sequences of tatD960 and tatD825 were searched for the presence of conserved domains. SignalP 4.1 server (http://www.cbs.dtu.dk/services/SignalP-4.1/) was used to check for the presence of signal peptides in TpTatDs. MEGA5 software was used to infer a phylogenetic tree of 53 DNases, including TatD DNases and some extracellular nucleases, from 
different bacteria. TatD amino acid sequences from S. aureus, Bacillus anthracis, S. pneumoniae, E. coli and $T$. pyogenes were aligned by Clustal W (https://www.genome.jp/tools-bin/clustalw) and presented by ESPript 3.0 (http://espript.ibcp.fr/ESPript/cgi-bin/ESPript.cgi). The identity rate for multiple sequence alignment of TatD DNase amino acid sequences was analyzed by DNAMAN software. Putative structures of TatD DNase 960 and TatD DNase 825 were predicted using SWISSMODEL (https://swissmodel.expasy.org/interactive) and drawn using PyMOL. The crucial functional residues were identified using the 3DLigandSite prediction server (http://www.sbg.bio.ic.ac.uk/3dligandsite/) with similar structures.

\subsection{Expression and purification of $T$. pyogenes TpTatD proteins}

The sequences encoding T. pyogenes TatD 960 and TatD 825 were PCR amplified from BMH06-3 genomic DNA using PrimeSTAR HS (Premix) (TAKARA, Dalian, China). PCR amplification was carried out in a $50 \mu \mathrm{L}$ reaction mixture containing each primer $(0.1 \mathrm{mM}), 25 \mu \mathrm{L}$ of PrimeSTAR HS (Premix) (TAKARA), and $100 \mathrm{ng}$ of template DNA. The thermal cycling conditions were identical for both primers: 30 cycles of $10 \mathrm{sec}$ at $98{ }^{\circ} \mathrm{C}, 5 \mathrm{sec}$ at $68^{\circ} \mathrm{C}$ and $1 \mathrm{~min}$ at $72^{\circ} \mathrm{C}$. pET28a-tatD960 was constructed using the restriction enzymes HindIII and NdeI, and the tatD 960 amplicon was cloned into the digested expression vector pET28a. The same approach was used to construct pET28a-tatD825 with the restriction enzymes BamHI and HindIII. The recombinant vectors were transformed into $E$. coli strain Trans-1-T1 (TransGen Biotech). The recombinant vectors were identified by PCR, restriction enzyme analysis and nucleotide sequencing.

The recombinant vectors were transformed into E. coli strain BL21 (DE3) (TransGen Biotech) for expression. His-tagged recombinant TatD proteins were expressed in E. coli. The soluble proteins were purified by affinity chromatography column using His Gravi Trap (GE Healthcare, Uppsala, Sweden). The purified protein was evaluated by SDS-PAGE and Western blot analysis. Briefly, the recombinant protein was electrophoresed on $12 \%$ SDS-PAGE gels and was then transferred to $0.45 \mu \mathrm{m}$ polyvinylidene fluoride using the wet transfer method. Rabbit polyclonal anti- $6 \times$ His $\operatorname{IgG}(1: 2,000)$ (BBI LIFE SCIENCE, Shanghai, China) was used as the primary antibody, and AP-labeled goat antirabbit IgG $(1: 5,000)$ (BBI LIFE SCIENCE) was used as the secondary antibody. PVDF membranes were incubated in BCIP/NBT solution for $5 \mathrm{~min}$, and images were acquired with a camera.

\subsection{Analysis of the DNase activity of TpTatDs}

Genomic DNA for this experiment was extracted from mouse livers using a TIANamp Genomic DNA Kit (TIANGEN Biotech, Beijing, China) according to the manufacturer's protocol. The pBR322 plasmid $(1 \mu \mathrm{g} / \mathrm{mL})$ was purchased from TAKARA Biomedical Technology Co., Ltd. (Dalian, China). The concentration of TpTatDs was quantified using a BCA Protein Assay Kit (Abcam, Cambridge, UK). DNA was incubated with TpTatDs (final concentrations: 0.5, 1, 2, 3, 4 and $5 \mu \mathrm{M}$ ) in PBS buffer $(\mathrm{pH}=7.4)$ in a final volume of $20 \mu \mathrm{L}$ at $37^{\circ} \mathrm{C}$ for $1 \mathrm{~h}$. PBS buffer was used as a negative control. The reaction time was $5,15,30,45$ or $60 \mathrm{~min}$. Six divalent metal ions were added to the reactions to test the facilitators of divalent metal ions. The influence of the concentration of $\mathrm{Mg}^{2+}$ (final concentrations: $0,2,4,6,8$ and $10 \mathrm{mM}$ ) on the function of TatD was investigated. To investigate the effect of TatD on circular DNA, plasmid pBR322 was cultured with TatDs at $37^{\circ} \mathrm{C}$ for $1 \mathrm{~h}$. The cultured mixture was analyzed using $1.0 \%$ agarose gel electrophoresis to determine whether the circular DNA was hydrolyzed.

The DNase activity of TpTatDs was quantitatively analyzed using PicoGreen reagent to measure the fluorescence intensity. The assay is based on the ability of PicoGreen reagent to bind to doublestranded DNA to enhance its fluorescence. The DNA substrate (200 ng) was incubated with TpTatDs 
$(2 \mu \mathrm{M})$ at different temperatures or $\mathrm{pH}$ values for 20 min prior to the addition of PBS buffer $(\mathrm{pH}=7.4)$ to bring the volume to $100 \mu \mathrm{L}$. PicoGreen working reagent was prepared by making a 1:200 dilution in TE buffer $(\mathrm{pH}=7.5)$. The reaction mixture was incubated with $100 \mu \mathrm{L}$ of PicoGreen working reagent at $25{ }^{\circ} \mathrm{C}$ for $5 \mathrm{~min}$. Two hundred microliter reaction systems were prepared in a 96-well fluorescence microtiter plate, and the fluorescence of the samples was measured using a VICTOR Nivo Multimode Plate Reader (PerkinElmer, Waltham, USA) at excitation and emission wavelengths of 480 and 520 $\mathrm{nm}$, respectively. The activity of TatD DNase was calculated using the following equation: DNase activity $(\%)=[($ fluorescence of the DNA control - fluorescence of the treated sample $) /$ fluorescence of the DNA control] $\times 100$ [24].

\subsection{Molecular docking analysis of luteolin and TpTatDs}

The molecular simulation software package Syby17.3 was used to generate the initial structure of luteolin, and the geometric structure of luteolin was optimized with the Tripos force field and Gasteiger-Marsili charge. Molecular docking analysis of TatD DNases and luteolin was carried out by AutoDock4.2 software. The Lamarckian genetic algorithm (LGA) was used to calculate the possible conformations of drug molecules that bind to the proteins. In the docking analysis, a maximum of 10 conformations of the compounds were considered, and the conformations with the lowest binding affinity were used for further analysis.

\subsection{Molecular interaction analysis of luteolin and TpTatDs}

The Biacore T200 system (GE Healthcare, Uppsala, Sweden) was used to measure the surface plasmon resonance (SPR) spectrum using an NTA sensor chip (GE Healthcare) with immobilized TatD DNase 960 and TatD DNase 825. Various concentrations of luteolin (3.125-50 $\mu \mathrm{M})$ in PBS-P buffer were injected at a flow rate of $30 \mu \mathrm{L} / \mathrm{min}$ for $3 \mathrm{~min}$ at $\mathrm{pH}=7.4$. The degree of luteolin binding to these TatD DNases was determined by measuring the SPR signal at the end of the dissociation phase. The rate constants, affinities and kinetics of the interaction between TatD DNases and luteolin were calculated by Trace Drawer (SPR Navi). The SPR Navi data viewer software extracted data before calculation in Trace Drawer.

\subsection{Gel assay of luteolin-TpTatD interactions}

Our previous research showed that the minimum inhibitory concentration (MICs) of luteolin against isolates of $T$. pyogenes is $78 \mu \mathrm{g} / \mathrm{mL}$ [33]. TatD DNases $(2 \mu \mathrm{M})$ and different MICs (final concentrations: $0,1 / 8,1 / 4,1 / 2,1,2$ and 4 MIC) of luteolin were added to Eppendorf tubes and supplemented with PBS ( $\mathrm{pH}=7.4$ ) to $20 \mu \mathrm{L}$. The mixtures were incubated at $37^{\circ} \mathrm{C}$ for $30 \mathrm{~min}$. Genomic DNA from mouse livers was extracted with phenol/chloroform. Two hundred nanograms of DNA from mouse livers was added to $20 \mu \mathrm{L}$ reaction systems and incubated at $37^{\circ} \mathrm{C}$ for an hour. A $20 \mu \mathrm{L}$ system containing only $200 \mathrm{ng}$ of DNA in PBS buffer was used as a control. Then, the mixtures were electrophoresed on a $0.8 \%$ agarose gel at a constant voltage of $90 \mathrm{~V}$ for an hour and analyzed.

\subsection{Statistical analysis}

All assays were carried out in triplicate. The data are presented as the mean \pm standard deviation (SD) values. Statistical analysis was performed using GraphPad Prism 5.0. 


\section{$5 \quad$ Funding}

This study was supported by grants from the National Natural Science Foundation of China (No. 31972736), the National Key Research and Development Plan (No. 2016YFD0501309).

\section{SUPPLEMENTARY FIGURES}

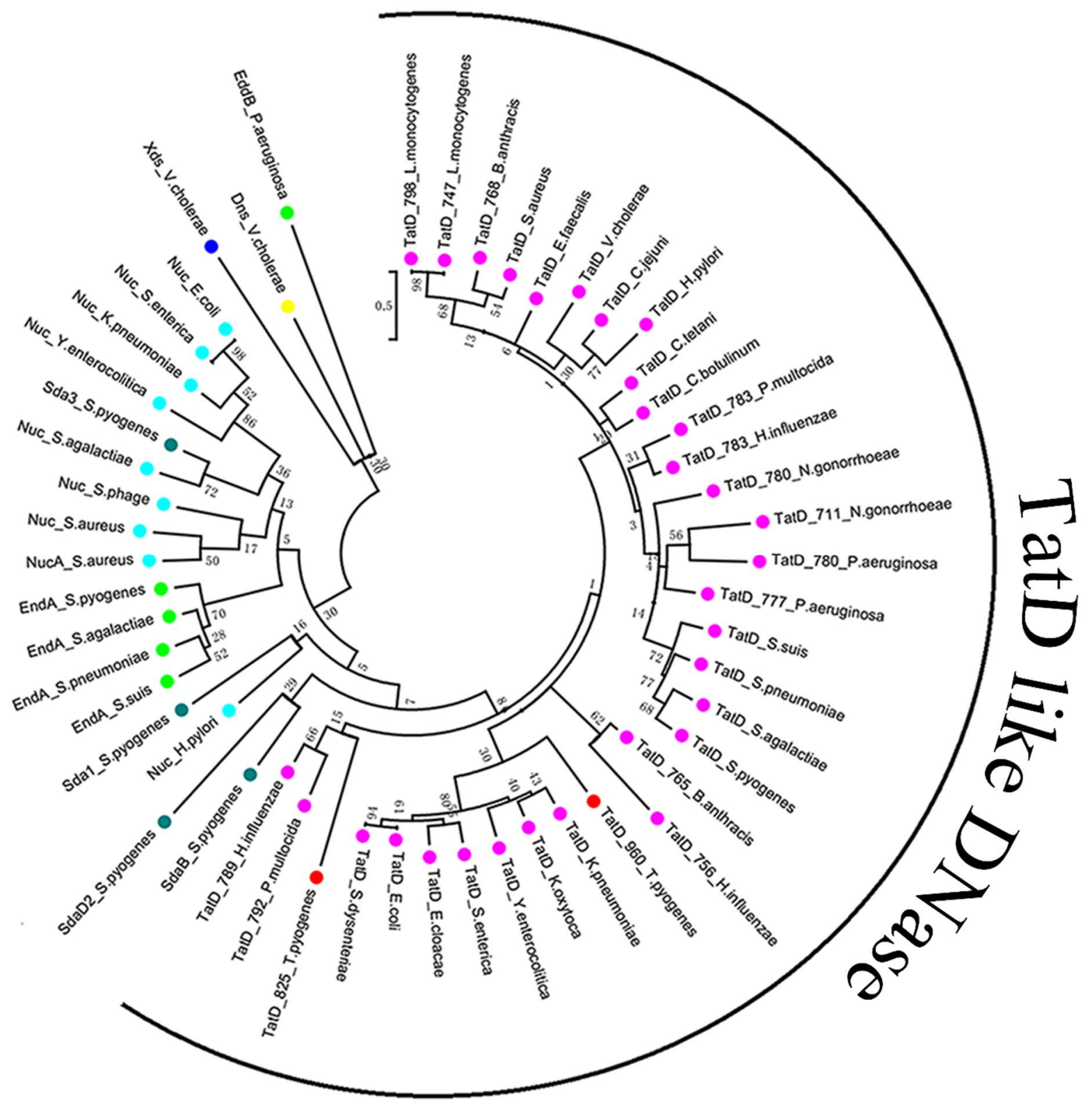

Figure S1. Phylogenetic tree of 53 DNases from bacteria constructed using maximum likelihood method. The DNases of bacteria are represented in different color shapes. TatD DNases of T. pyogenes are highlighted in red. 

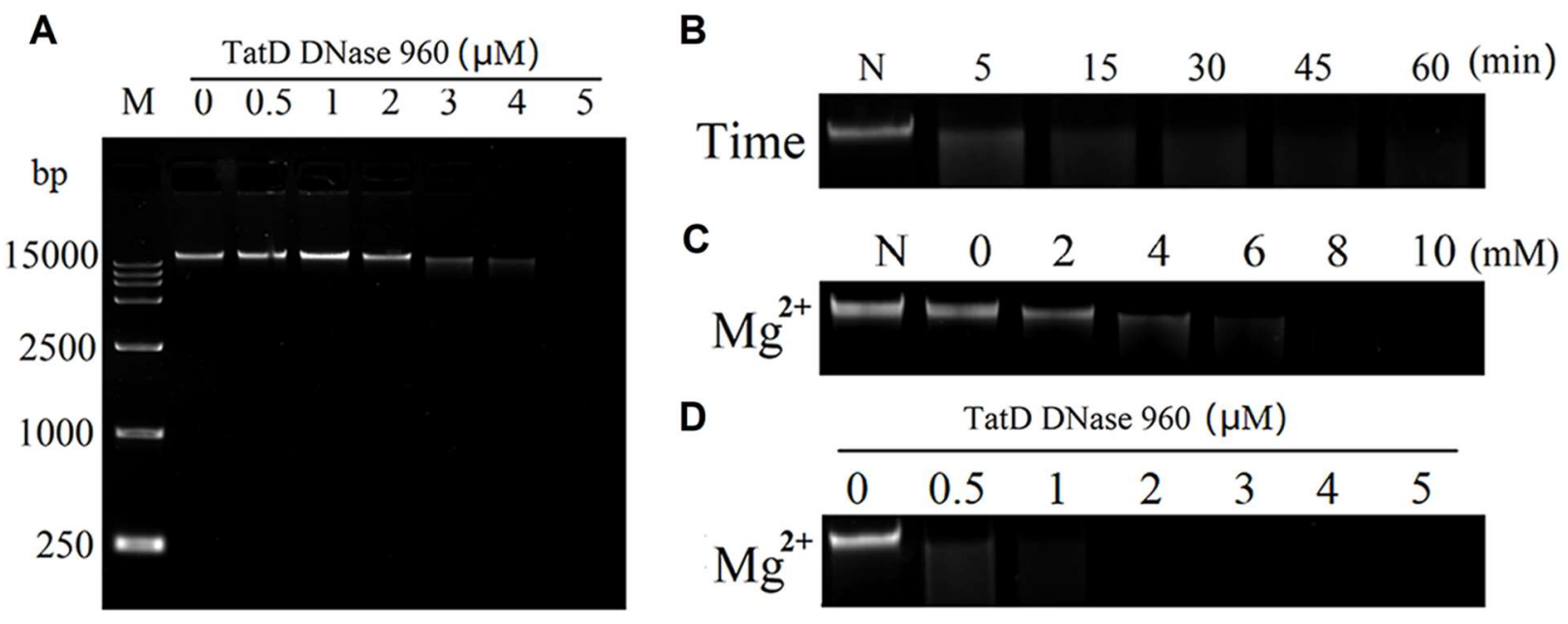

Figure S2. $\mathrm{Mg}^{2+}$-dependent DNase activity of TatD DNase 960. (A) TpTatD 960 can weakly degrade DNA. TpTatD 960 at various concentrations $(0-5 \mu \mathrm{M})$ was incubated with DNA (200 ng) in PBS buffer $(\mathrm{pH}=7.4)$ at $37{ }^{\circ} \mathrm{C}$ for $1 \mathrm{~h}$. (B) TpTatD $960(5 \mu \mathrm{M})$ was able to hydrolyze DNA completely within $1 \mathrm{~h}$. (C) TatD 960 is a $\mathrm{Mg}^{2+}$-dependent DNase. DNA was completely hydrolyzed incubating with TpTatD $960(2 \mu \mathrm{M})$ and $\mathrm{Mg}^{2+}(8 \mathrm{mM})$ at $37^{\circ} \mathrm{C}$ for $1 \mathrm{~h}$. (D) $\mathrm{Mg}^{2+}$ can promote the hydrolysis of DNA by TatD DNase 960. TpTatD $960(1 \mu \mathrm{M})$ with $\mathrm{Mg}^{2+}(10 \mathrm{mM})$ was able to hydrolyze DNA completely for $1 \mathrm{~h}$.
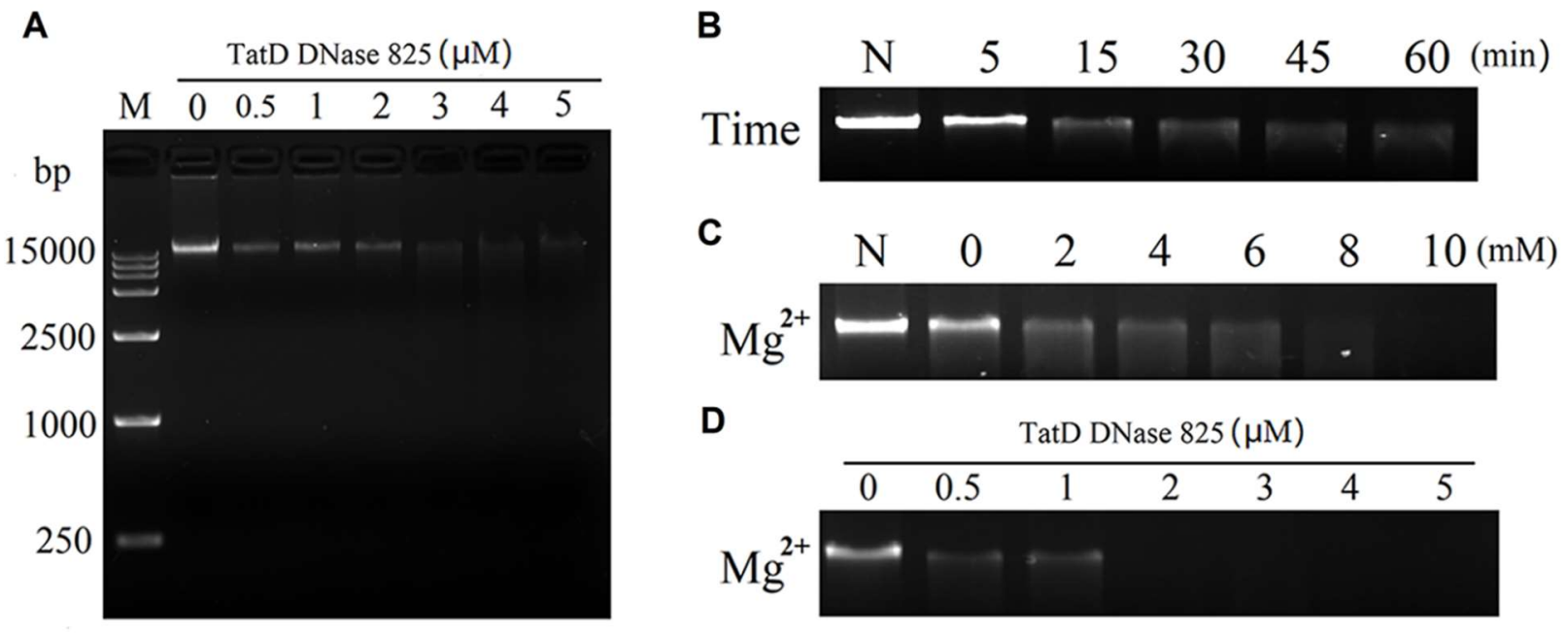

Figure S3. $\mathrm{Mg}^{2+}$-dependent DNase activity of TatD DNase 825. (A) TpTatD 825 can weakly degrade DNA. TpTatD 825 at various concentrations $(0-5 \mu \mathrm{M})$ was incubated with DNA (200 ng) as Supplementary Figure 2a. (B) TpTatD $825(5 \mu \mathrm{M})$ was able to hydrolyze most of DNA within $1 \mathrm{~h}$. (C) TpTatD 825 is a $\mathrm{Mg}^{2+}$-dependent DNase. DNA was completely hydrolyzed incubating with TpTatD $825(2 \mu \mathrm{M})$ and $\mathrm{Mg}^{2+}(10 \mathrm{mM})$ at $37{ }^{\circ} \mathrm{C}$ for $1 \mathrm{~h}$. (D) $\mathrm{Mg}^{2+}$ can promote the hydrolysis of DNA by TpTatD 825 . TpTatD $825(2 \mu \mathrm{M})$ with $\mathrm{Mg}^{2+}(10 \mathrm{mM})$ was able to hydrolyze DNA completely for $1 \mathrm{~h}$. 


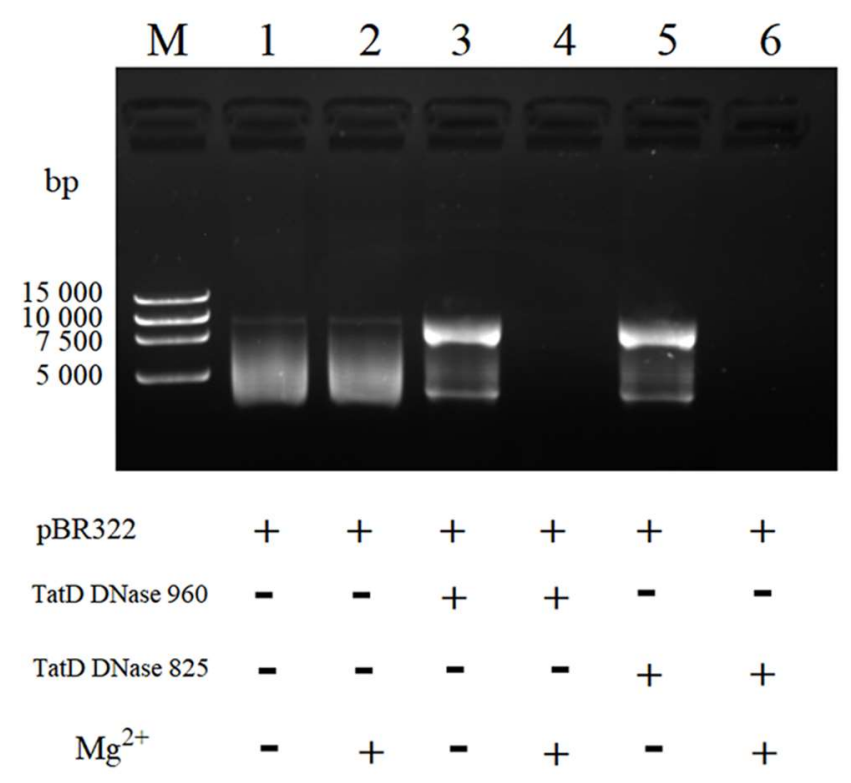

Figure S4. The ability of TpTatDs to digest plasmid DNA substrates. $200 \mathrm{ng}$ plasmid pBR322 was incubated with $2 \mu \mathrm{M}$ TpTatD 960/TpTatD 825 at $37{ }^{\circ} \mathrm{C}$ for $1 \mathrm{~h}$, respectively. $\mathrm{Mg}^{2+}$ was added to the complex as a stimulator, and the final concentration of the $\mathrm{Mg}^{2+}$ was $10 \mathrm{mM}$. The DNA was visualized by agarose gel electrophoresis. TatD DNases can hydrolyze plasmid pBR322 without the catalysis of metal ions, but the hydrolysis ability is weak. $\mathrm{Mg}^{2+}$ can promote TatD DNases to hydrolyze pBR322.

A
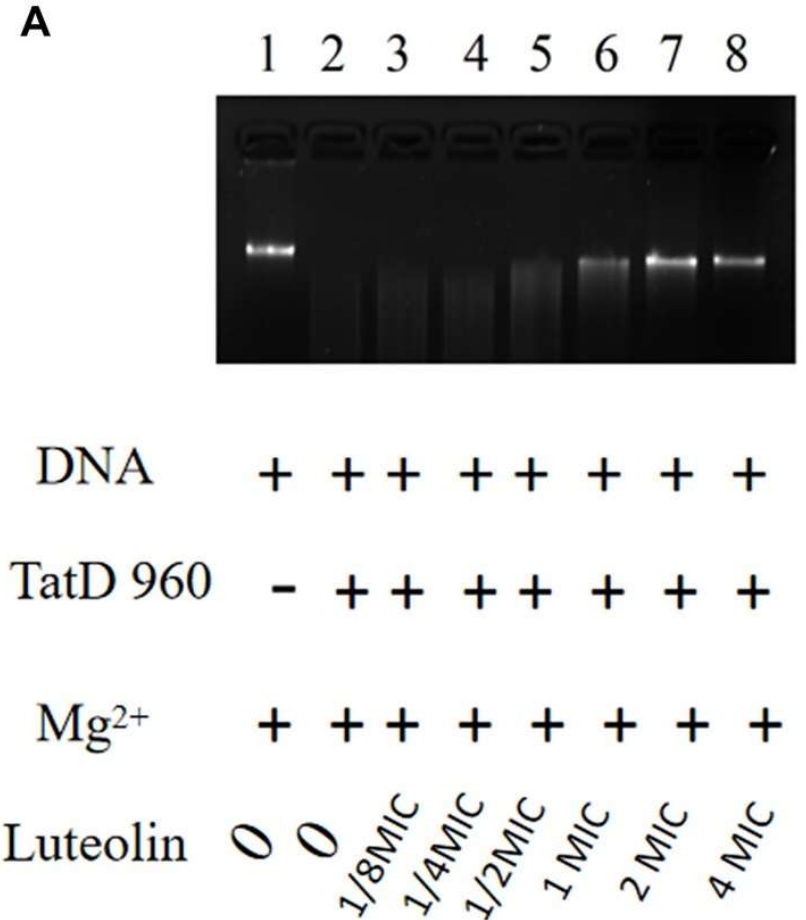

B
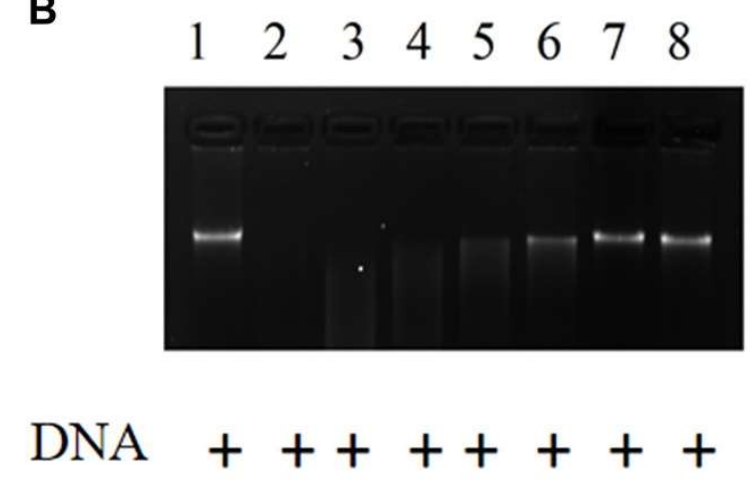

TatD $825-+++++++$

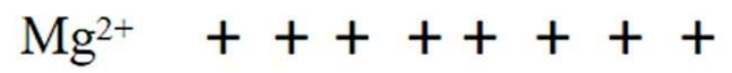

Luteolin

Figure S5. The effect of luteolin on DNase activity of TpTatDs. Luteolin can inhibit the ability of TatD DNases to hydrolyze DNA. $2 \mu \mathrm{M}$ TatD DNases treated by different concentrations of luteolin (final concentrations: $0,1 / 8,1 / 4,1 / 2,1,2$ and 4 MIC). The complex was incubated with 200 ng linear DNA and $\mathrm{Mg}^{2+}(10 \mathrm{mM})$ at $37^{\circ} \mathrm{C}$ for $1 \mathrm{~h}$. (A) The effect of luteolin on DNase activity of TpTatD 960; (B) The effect of luteolin on DNase activity of TpTatD 825. 


\section{$7 \quad$ References}

1. Ribeiro, M.G.; Risseti, R.M.; Bolaños, C.A.; Caffaro, K.A.; de Morais, A.C.; Lara, G.H.; Zamprogna, T.O.; Paes, A.C.; Listoni, F.J.; Franco, M.M. Trueperella pyogenes multispecies infections in domestic animals: a retrospective study of 144 cases (2002 to 2012). Vet. Q. 2015, 35, 82-87. doi: 10.1080/01652176.2015.1022667.

2. Ochi, K.; Okamoto, M.; Okamoto, M.; Okura, M.; and Takamatsu, D. Development of a novel Trueperella pyogenes-specific PCR assay. J. Vet. Med. Sci. 2020, 82, 109-114. doi: 10.1292/jvms.19-0522.

3. Santos, T.M.; Caixeta, L.S.; Machado, V.S.; Rauf, A.K.; Gilbert, R.O.; Bicalho, R.C. Antimicrobial resistance and presence of virulence factor genes in Arcanobacterium pyogenes isolated from the uterus of postpartum dairy cows. Vet. Microbiol. 2010, 145, 84-89. doi: 10.1016/j.vetmic.2010.03.001.

4. Bicalho, M.L.; Lima, F.S.; Machado, V.S.; Meira, E.B.Jr.; Ganda, E.K.; Foditsch, C.; Bicalho, R.C.; Gilbert, R.O. Associations among Trueperella pyogenes, endometritis diagnosis, and pregnancy outcomes in dairy cows. Theriogenology. 2016, 85, 267-274. doi: 10.1016/j.theriogenology.2015.09.043.

5. Zhang, D.; Zhao, J.; Wang, Q.; Liu, Y.; Tian, C.; Zhao, Y.; Yu, L.; Liu, M. Trueperella pyogenes isolated from dairy cows with endometritis in Inner Mongolia, China: Tetracycline susceptibility and tetracycline-resistance gene distribution. Microb. Pathog. 2017, 105, 51-56. doi: 10.1016/j.micpath.2017.02.010.

6. Ishiyama, D.; Mizomoto, T.; Ueda, C.; Takagi, N.; Shimizu, N.; Matsuura, Y.; Makuuchi, Y.; Watanabe, A.; Shinozuka, Y.; Kawai, K. Factors affecting the incidence and outcome of Trueperella pyogenes mastitis in cows. J. Vet. Med. Sci. 2017, 79, 626-631. doi: 10.1292/jvms.160401

7. Risseti, R.M.; Zastempowska, E.; Twarużek, M.; Lassa, H.; Pantoja, J.C.F.; de Vargas, A.P.C; Guerra, S.T.; Bolaños, C.A.D.; de Paula, C.L.; Alves, A.C.; et al. Virulence markers associated with Trueperella pyogenes infections in livestock and companion animals. Lett. Appl. Microbiol. 2017, 65, 125-132. doi: 10.1111/lam.12757.

8. Funke, G.; von Graevenitz, A.; Clarridge, J. E.; Bernard, K. A. Clinical microbiology of coryneform bacteria. Clin. Microbiol. Rev. 1997, 10, 125-159. doi: 10.1128/CMR.10.1.125159.1997.

9. Lynch, M.; O’Leary, J.; Murnaghan, D.; Cryan, B. Actinomyces pyogenes septic arthritis in a diabetic farmer. J. Infect. 1998, 37, 71-73. doi: 10.1016/s0163-4453(98)90862-3.

10. Kavitha, K.; Latha, R.; Udayashankar, C.; Jayanthi, K.; Oudeacoumar, P. Three cases of Arcanobacterium pyogenes-associated soft tissue infection. J. Med. Microbiol. 2010, 59, 736-739. doi: 10.1099/jmm.0.016485-0. 
11. Hermida Amejeiras, A.; Romero Jung, P.; Cabarcos Ortiz De Barrón, A.; Treviño Castallo, M. One case of pneumonia with Arcanobacterium pyogenes. An. Med. Int. 2004, 21, 334-336. doi: 10.4321/s0212-71992004000700006.

12. Levy, C.E.; Pedro, R.J.; von Nowakonski, A.; Holanda, L.M.; Brocchi, M.; Ramo, M.C. Arcanobacterium pyogenes sepsis in farmer. Brazil. Emerg. Infect. Dis. 2009, 15, 1131-1132. doi: 10.3201/eid1507.081072.

13. Semaan, A.; Tayeh, G.A.; Chebel, J.A.; Hallit, R.; Matta, M.; Hajj, P. Arcanobacterium pyogenes and encrusted pyelitis. Future. Sci. OA. 2019, 6, FSO430. doi: 10.2144/fsoa-2019-0021.

14. Deliwala, S.; Beere, T.; Samji, V.; Mcdonald, P.J.; Bachuwa, G. When Zoonotic Organisms Cross Over-Trueperella pyogenes Endocarditis Presenting as a Septic Embolic Stroke. Cureus. 2020, 12, e7740. doi: 10.7759/cureus.7740.

15. Rzewuska, M.; Kwiecień, E.; Chrobak-Chmiel, D.; Kizerwetter-Świda, M.; Stefańska, I.; Gieryńska, M. Pathogenicity and Virulence of Trueperella pyogenes: A Review. Int J Mol Sci. 2019,20, 2737. doi: 10.3390/ijms20112737.

16. Jarosz, L.S.; Gradzki, Z.; Kalinowski, M. Trueperella pyogenes infections in swine: Clinical course and pathology. Pol. J. Vet. Sci. 2014, 17, 395-404. doi: 10.2478/pjvs-2014-0055.

17. Wexler, M.; Sargent, F.; Jack, R.L.; Stanley, N.R.; Bogsch, E.G.; Robinson, C.; Berks, B.C.; Palmer, T. TatD is a cytoplasmic protein with DNase activity. No requirement for TatD family proteins in sec-independent protein export. J. Biol. Chem. 2000, 275, 16717-16722. doi: 10.1074/jbc.M000800200.

18. Chen, Y.C.; Li, C.L.; Hsiao, Y.Y.; Duh, Y.; Yuan, H.S. Structure and function of TatD exonuclease in DNA repair. Nucleic. Acids. Res. 2014, 42, 10776-10785. doi: 10.1093/nar/gku732.

19. Matos, C.F.; Di Cola, A.; Robinson, C. TatD is a central component of a Tat translocon-initiated quality control system for exported FeS proteins in Escherichia coli. EMBO. Rep. 2009, 10, 474479. doi: 10.1038/embor.2009.34.

20. Gannavaram, S.; Debrabant, A. Involvement of TatD nuclease during programmed cell death in the protozoan parasite Trypanosoma brucei. Mol. Microbiol. 2012, 83, 926-935. doi: 10.1111/j.1365-2958.2012.07978.x.

21. Chen, L.; Shen, D.; Sun, N.; Xu, J.; Wang, W.; Dou, D. Phytophthora sojae TatD nuclease positively regulates sporulation and negatively regulates pathogenesis. Mol. Plant. Microbe. Interact. 2014, 27, 1070-1080. doi: 10.1094/MPMI-05-14-0153-R.

22. Chang, Z.; Jiang, N.; Zhang, Y.; Lu, H.; Yin, J.; Wahlgren, M.; Cheng, X.; Cao, Y.; Chen, Q. The TatD-like DNase of Plasmodium is a virulence factor and a potential malaria vaccine candidate. Nat. Commun. 2016, 7, 11537. doi: 10.1038/ncomms11537.

23. Zhang, K.; Jiang, N.; Chen, H.; Zhang, N.; Sang, X.; Feng, Y.; Chen R; Chen Q. TatD DNases of African trypanosomes confer resistance to host neutrophil extracellular traps. Sci. China. Life. Sci. 2021, 64, 621-632. doi: 10.1007/s11427-020-1854-2. 
24. Jhelum, H.; Sori, H.; Sehgal, D. A novel extracellular vesicle-associated endodeoxyribonuclease helps Streptococcus pneumoniae evade neutrophil extracellular traps and is required for full virulence. Sci. Rep. 2018, 8, 7985. doi: 10.1038/s41598-018-25865-z.

25. Zhang, T.; Qiu, Y.; Luo, Q.; Zhao, L.; Yan, X.; Ding, Q.; Jiang, H.; Yang, H. The Mechanism by Which Luteolin Disrupts the Cytoplasmic Membrane of Methicillin-Resistant Staphylococcus aureus. J. Phys. Chem. B. 2018, 122, 1427-1438. doi: 10.1021/acs.jpcb.7b05766.

26. Usman Amin, M.; Khurram, M.; Khan, T.A.; Faidah, H.S.; Ullah Shah, Z.; Ur Rahman, S.; Haseeb, A.; Ilyas, M.; Ullah, N.; Umar Khayam, S.M.; et al. Effects of Luteolin and Quercetin in Combination with Some Conventional Antibiotics against Methicillin-Resistant Staphylococcus aureus. Int. J. Mol. Sci. 2016, 17, 1947. doi: 10.3390/ijms17111947.

27. Siriwong, S.; Thumanu, K.; Hengpratom, T.; Eumkeb, G. Synergy and Mode of Action of Ceftazidime plus Quercetin or Luteolin on Streptococcus pyogenes. Evid. Based. Complement. Alternat. Med. 2015, 759459. doi: 10.1155/2015/759459.

28. Qian, W.; Fu, Y.; Liu, M.; Zhang, J.; Wang, W.; Li, J.; Zeng, Q.; Wang, T.; Li, Y. Mechanisms of Action of Luteolin Against Single and Dual-Species of Escherichia coli and Enterobacter cloacae and Its Antibiofilm Activities. Appl. Biochem. Biotechnol. 2021, 193, 1397-1414. doi:10.1007/s12010-020-03330-w.

29. Eumkeb, G.; Siriwong, S.; Thumanu, K. Synergistic activity of luteolin and amoxicillin combination against amoxicillin-resistant Escherichia coli and mode of action. J. Photochem. Photobiol. B. 2012, 117, 247-253. doi: 10.1016/j.jphotobiol.2012.10.006.

30. Rivera, M.L.C.; Hassimotto, N.M.A.; Bueris, V.; Sircili, M.P.; de Almeida, F.A.; Pinto, U.M. Effect of Capsicum Frutescens Extract, Capsaicin, and Luteolin on Quorum Sensing Regulated Phenotypes. J. Food. Sci. 2019, 84, 1477-1486. doi: 10.1111/1750-3841.14648.

31. Qiu, j.; Li, H.; Meng, H.; Hu, C.; Li, J.; Luo, M.; Dong, J.; Wang, X.; Wang, J.; Deng, Y.; et al. Impact of luteolin on the production of alpha-toxin by Staphylococcus aureus. Lett. Appl. Microbiol. 2011, 53, 238-243. doi: 10.1111/j.1472-765X.2011.03098.x.

32. Shen, X.F.; Ren, L.B.; Teng, Y.; Zheng, S.; Yang, X.L.; Guo, X.J.; Wang, X.Y.; Sha, K.H.; Li, N.; Xu, G.Y.; et al. Luteolin decreases the attachment, invasion and cytotoxicity of UPEC in bladder epithelial cells and inhibits UPEC biofilm formation. Food. Chem. Toxicol. 2014, 72, 204211. doi: 10.1016/j.fct.2014.07.019.

33. Guo, Y.; Liu, Y.; Zhang, Z.; Chen, M.; Zhang, D.; Tian, C.; Liu, M.; Jiang, G. The Antibacterial Activity and Mechanism of Action of Luteolin Against Trueperella pyogenes. Infect. Drug. Resist. 2020, 13, 1697-1711. doi: 10.2147/IDR.S253363.

34. Zhang, D.; Gao, X.; Song, X.; Zhou, W.; Hong, W.; Tian, C.; Liu, Y.; Liu, M. Luteolin Showed a Resistance Elimination Effect on Gentamicin by Decreasing MATE mRNA Expression in Trueperella pyogenes. Microb. Drug. Resist. 2019, 25, 619-626. doi: 10.1089/mdr.2018.0097. 
35. Zhou, Y.; Xiao, B.; Jiang, N.; Sang, X.; Yang, N.; Feng, Y.; Jiang, L.; Chen, Q. Expression and functional analysis of the TatD-like DNase of Plasmodium knowlesi. Parasit. Vectors. 2018, 11, 629. doi: 10.1186/s13071-018-3251-4.

36. Lee, K.Y.; Cheon, S.H.; Kim, D.G.; Lee, S.J.; Lee, B.J. A structural study of TatD from Staphylococcus aureus elucidates a putative DNA-binding mode of a $\mathrm{Mg}^{2+}$-dependent nuclease. IUCrJ. 2020, 7: 509-521. doi: 10.1107/S2052252520003917.

37. Singh, D.; Rahi, A.; Kumari, R.; Gupta, V.; Gautam, G.; Aggarwal, S.; Rehan, M.; Bhatnagar, R. Computational and mutational analysis of TatD DNase of Bacillus anthracis. J. Cell. Biochem. 2019. doi: $10.1002 /$ jcb.28408. Online ahead of print.

38. Russo, M.; Moccia, S.; Spagnuolo, C.; Tedesco, I.; Russo, G.L. Roles of flavonoids against coronavirus infection. Chem. Biol. Interact. 2020, 328, 109211. doi: 10.1016/j.cbi.2020.109211.

39. Yu, R.; Chen, L.; Lan, R.; Shen, R.; Li, P. Computational screening of antagonists against the SARS-CoV-2 (COVID-19) coronavirus by molecular docking. Int J Antimicrob Agents. 2020, 56, 106012. doi: 10.1016/j.ijantimicag.2020.106012.

40. Zima, V.; Radilová, K.; Kožíšek, M.; Albiñana, CB.; Karlukova, E.; Brynda, J.; Fanfrlík, J.; Flieger, M.; Hodek, J.; Weber, J.; et al. Unraveling the anti-influenza effect of flavonoids: Experimental validation of luteolin and its congeners as potent influenza endonuclease inhibitors. Eur. J. Med. Chem. 2020, 208, 112754. doi: 10.1016/j.ejmech.2020.112754.

41. Gangadharappa, B.S.; Sharath, R.; Revanasiddappa, P.D.; Chandramohan, V.; Balasubramaniam, M.; Vardhineni, T.P. Structural insights of metallo-beta-lactamase revealed an effective way of inhibition of enzyme by natural inhibitors. J. Biomol. Struct. Dyn. 2020, 38, 3757-3771. doi: 10.1080/07391102.2019.1667265.

42. Zhou, Y.; Huang, L.; Ji, S.; Hou, S.; Luo, L.; Li, C.; Liu, M.; Liu, Y.; Jiang, L. Structural Basis for the Inhibition of the Autophosphorylation Activity of HK853 by Luteolin. Molecules. 2019, 24, 933. doi: 10.3390/molecules24050933.

43. Zuo, R.; de Oliveira, A.; Bullita, E.; Torino, M.I.; Padgett-Pagliai, K.A.; Gardner, C.L.; Harrison, N.A.; da Silva, D.; Merli, M.L.; Gonzalez, C.F.; et al. Identification of flavonoids as regulators of YbeY activity in Liberibacter asiaticus. Environ. Microbiol. 2019, 21, 4822-4835. doi: $10.1111 / 1462-2920.14831$. 